\title{
Pertrochantäre Femurfraktur: Intraoperativer Wechsel von intra- auf extramedulläres Implantat
}

\author{
Joachim Windolf
}

Die 48-jährige, adipöse Monika R. war auf dem Weg zur Arbeit als Passantin von einem Pkw angefahren worden und hatte sich hierbei eine pertrochantäre Femurfraktur rechts zugezogen (Abb.1). Die Verletzung wurde als 31-A1.3-Fraktur klassifiziert und sollte am Unfalltag als Ausbildungseingriff mit einem intramedullären Kraftträger (PFN-A) stabilisiert werden.

Bereits die Lagerung der Patientin auf dem Extensionstisch war angesichts der Adipositas erheblich erschwert und benötigte über eine halbe Stunde Zeit. Schließlich ließ sich aber unter Bildwandlerkontrolle eine geschlossene Reposition der Fraktur mit anatomisch achsgerechter Stellung der Fragmente erzielen. Angesichts der erschwerten Bedingungen übernahm allerdings der verantwortliche Facharzt bereits nach der Lagerung den Eingriff. Der Zugang erfolgte über eine $10 \mathrm{~cm}$ lange Längsinzision ca. $5 \mathrm{~cm}$ oberhalb des gerade eben tastbaren Trochanter major. Auch das Einbringen und exakte Platzieren des Führungsdrahts an der Spitze des Trochanters bedurfte aufgrund des erheblichen Umfangs des Subkutangewebes einiger Mühen. Schließlich konnte aber die Eröffnung des Femurs über den Führungsdraht erfolgen und der zuvor ausgemessene $240 \mathrm{~mm}$ lange und $10 \mathrm{~mm}$ dicke Nagel eingeführt werden. In der Bildwandlerkontrolle zeigte sich zunächst, dass der Nagel nicht tief genug eingebracht war, sodass nach neuerlichem Aufbohren der Nagel bis zur gewünschten Tiefe inseriert wurde. In der Bildwandlerkontrolle fand sich nun allerdings eine komplette Sprengung des Trochanter major in Längsrichtung mit grober Dislokation der Fraktur. Unter Leitung des hinzugerufenen Oberarztes folgten nun mehrere frustrane

OP-JOURNAL 2008; 24: 224-225

(c) Georg Thieme Verlag KG Stuttgart • New York DOI 10.1055/s-2008-1039127
Versuche, eine erneute Reposition zu erzielen und den Nagel zu inserieren. Hierdurch kam es aber zu einer weiteren Fragmentierung des Trochanter major. Schließlich wurde der intraoperative Befund dem Klinikleiter mit der Frage demonstriert, ob angesichts der aussichtslos anmutenden Situation ein Umstieg auf eine prothetische Versorgung erfolgen sollte.

Mittlerweile hatte der Eingriff bereits weit über 2 Stunden gedauert und die Inzision wies eine Länge von über $30 \mathrm{~cm}$ auf. Aufgrund des jungen Alters der Patientin sollte aber eine prothetische Versorgung vermieden werden, sodass der Klinikleiter den Eingriff selbst übernahm. Zunächst wurde die Trochanterregion nach erneuter Schnitterweiterung exploriert. Es zeigte sich eine komplette Fragmentierung des Trochanters und eine Verkippung des Kopf-HalsFragments mit einer ausgedehnten medialen Dehiszenz. Mit großer Mühe konnte schließlich in Abduktion, Beugung und Außenrotation der rechten Hüfte unter Valgisierung des Kopf-HalsFragments eine Reposition mit medialer Abstützung erzielt und durch den Einsatz von 2 großen Wellerzangen passager gehalten werden. Allerdings musste dabei eine Rotation mit vermehrter Anteversion in Kauf genommen werden. Da aufgrund der zerstörten Nageleintrittsstelle die Implantation eines PFN-A nicht mehr möglich war, wurde das Konzept geändert und die Stabilisierung mit einer DHS angestrebt. Aufgrund der nun extraanatomischen Situation und der erheblichen Adipositas gestaltete sich die exakte Platzierung des Zieldrahts überaus mühsam. Schließlich konnte der Draht platziert werden und hierüber die Implantation einer DHS mit einem CCD-Winkel von 150 Grad erfolgen (Abb. 2). Die intraoperative Bildgebung zeigte zwar eine grenzwertige Lage der DHS, aber ein akzeptables Repositionsergebnis mit medialer Abstützung, sodass auf eine weitere Ausdeh-

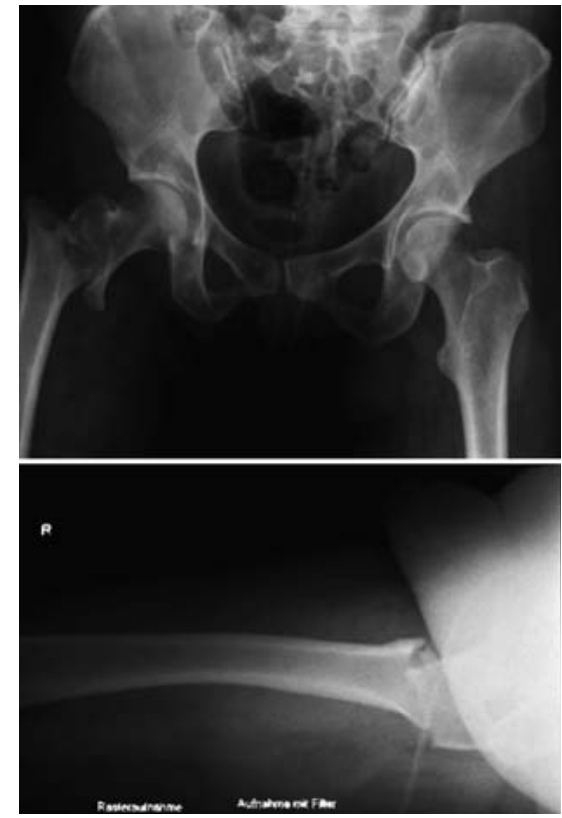

Abb. 1 Unfallaufnahmen.

nung des Eingriffs nach insgesamt fast 4-stündiger Dauer verzichtet wurde. Nach einer Jet-Lavage wurde die Wunde verschlossen.

Im postoperativen Verlauf ergaben sich zunächst keine Komplikationen. Die Patientin war beschwerdefrei und konnte die krankengymnastische Weiterbehandlung regelrecht aufnehmen. In der postoperativen Röntgenkontrolle zeigte sich ein dorsokraniales Ausschneiden der Schenkelhalsschraube aus dem Hüftkopf (Abb. 3). Angesichts der bestehenden medialen Abstützung und der klinischen Beschwerdefreiheit der Patientin wurde nach ausführlicher Aufklärung auf eine operative Revision verzichtet. Veilmehr wurde die schmerzadaptierte Teilbelastung der Extremität erlaubt.

Bei der klinischen und röntgenologischen Nachuntersuchung der Patientin 10 Wochen später zeigte sich eine zu- 

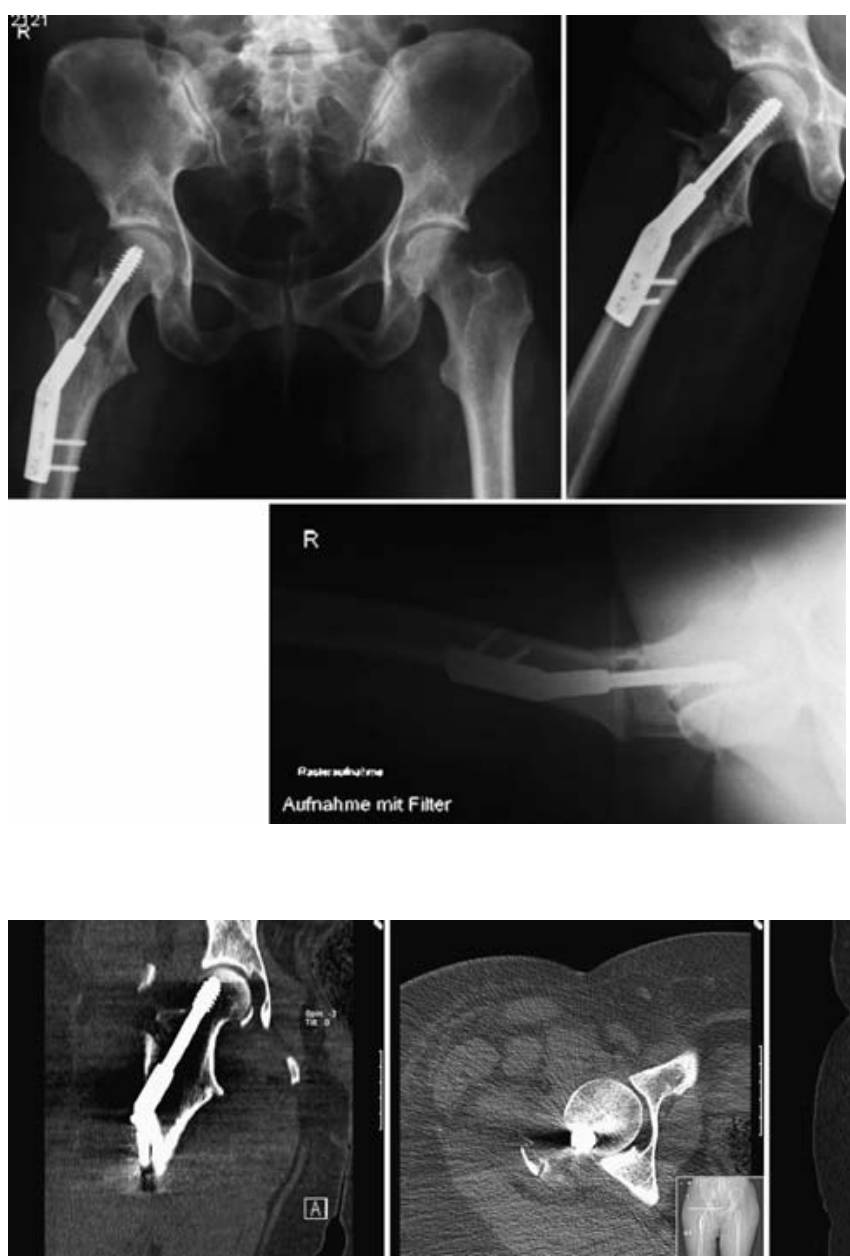

Abb. 3 CT-Kontrolle.
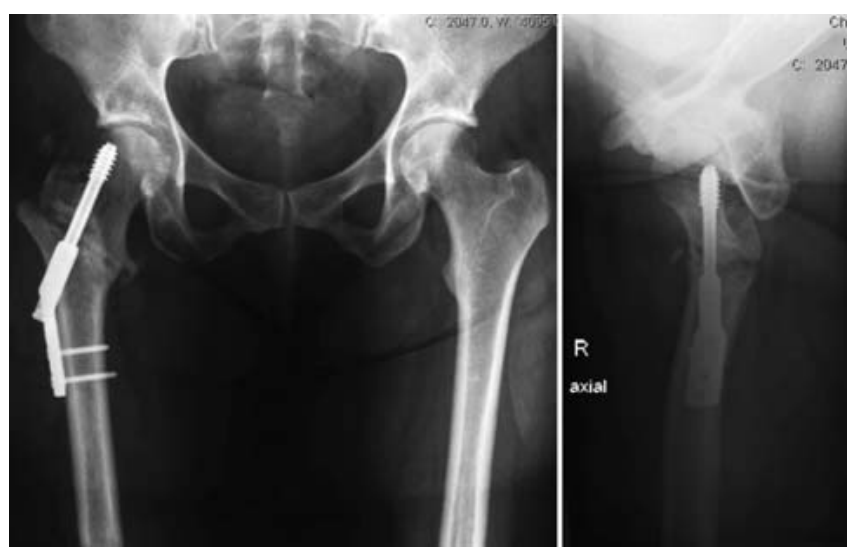

nehmende Durchbauung der Fraktur unter zunehmendem Belastungsaufbau (Abb. 4 und 5).

\section{Fazit für die Praxis}

Im vorliegenden Fall konnte der Wechsel auf ein extramedulläres Verfahren und eine valgisierende Umstellung des Kopf-Hals-Fragments den fehlgeschlagenen Versuch einer Marknagelosteo-

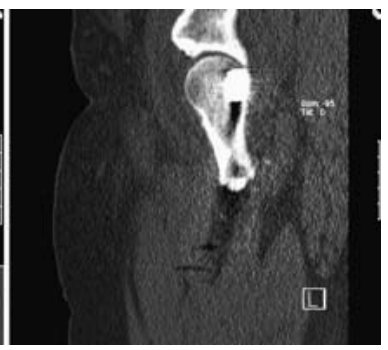

Abb. 4 Kontrolle nach 10 Wochen.

Abb. 2 Operatives Ergebnis.
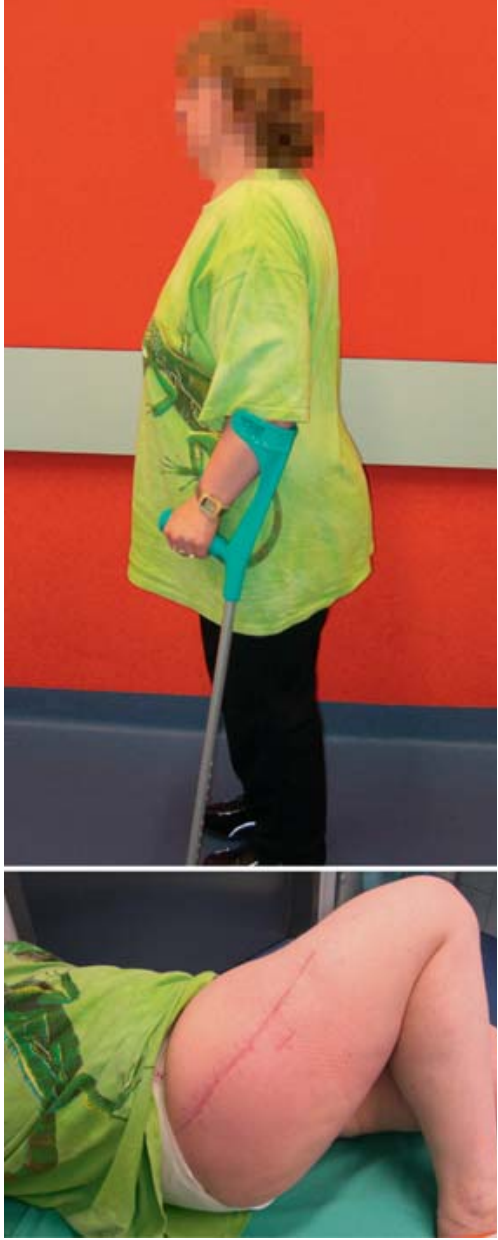

Abb. 5 Kontrolle nach 10 Wochen.

Prof. Dr. med. Joachim Windolf

synthese eine proximale Femurfraktur auffangen. Ein Umstieg auf eine prothetische Versorgung der Patientin kam aufgrund des Lebensalters nicht in Betracht. Postoperativ konnte die „windige" Osteosynthese durch Ruhe bewahren und vorsichtigen Belastungsaufbau zur Ausheilung der Fraktur geführt werden.
Direktor

Klinik für Unfall- und Handchirurgie Universitätsklinikum Düsseldorf Moorenstraße 5 40225 Düsseldorf

E-Mail: windolf@uni-duesseldorf.de 